\title{
Editorial
}

\section{Large vestibular aqueduct: large endolymphatic sac?}

\author{
P. D. PhelPS, M.D., F.R.C.S., F.R.C.R.
}

The first description of large vestibular aqueduct (LVA) was by Carlo Mondini in 1791 from his dissection of the temporal bones of a boy born deaf (Mondini, 1791) Mondini not only described and drew the LVA which he measured at seven lines or about $15 \mathrm{~mm}$ wide, but he opened the voluminous endolymphatic sac which he found to be 'full of a rather viscous humour, clear and yellowish which obviously flowed from the vestibule'. Mondini also noted that 'the bony plate was missing which protects the aqueduct of the vestibule like a scale whose uneven edge forms that chink which is clearly visible in the posterior face of the pyramid'. Mondini's precise and detailed account was of a congenital malformation of the inner ear consisting of a grossly enlarged vestibular aqueduct and endolymphatic sac and duct, a larger than normal vestibule, a normal basal turn of the cochlea, but with the distal 1.5 coils replaced by a sac; ('amplam caveam cavitati ultimi gyri respondentem;). Thus there can be no doubt as to what Mondini actually described but unfortunately the term 'Mondini's dysplasia' has been so grossly abused subsequently as to make it virtually meaningless.

The first description of LVA based on imaging was by Valvassori and Clemis in 1978 who showed LVA in 50 patients by polytomography and coined the description 'large vestibular aqueduct syndrome' when this was accompanied by sensorineural hearing loss. Subsequently, the ease with which an LVA can be demonstrated by axial computed tomography (CT) has meant that a plethora of accounts of LVA based on CT has been published, such as those of Emmett (1985) and Levenson et al. (1989). However in an account of another large series of LVA, Jackler and De La Cruz drew attention to the poor results of shunt operations to attempt 'stabilisation' of hearing (Jackler and De La Cruz, 1989).

In a previous editorial on LVA, I indicated the probable association with enlargement of the endolyphatic duct and sac (Phelps, 1994) and this has been borne out by histological studies firstly by Kodama and Sando in 1982 and Gussen in 1986. These Mondini-type deformities of cochlear and vestibular systems were perhaps surprisingly considered by these authors to be the result of pressure erosion of bone, although there was no evidence of Menièretype endolymphatic hydrops in the dissections.

Meantime, significant advances in magnetic resonance imaging (MRI) have meant that it is now possible with the latest thin section high resolution techniques to demonstrate the fluid-filled endolymphatic sac and duct. Moreover it would seem that enlargement of the vestibular aqueduct does not have any sort of constant relation to an enlarged endolymphatic duct and sac. In one series three cases (five inner ears) with enlarged bony vestibular aqueducts on CT showed no evidence of endolymphatic duct or sac enlargement on MRI. MR alone identified a single case of mild cochlear anomaly in conjunction with an enlarged endolymphatic duct and sac (Harnoberger et al., 1985). Another case report showed massive endolymphatic sacs on the MR study (Reussner et al., 1995).

A genetic association of deafness and goitre first described by Pendred exactly one hundred years ago is at present being intensively investigated at the Institute of Child Health in London (Pendred, 1896). The abnormal chromosome in this recessive condition has been confirmed recently, but it is the ear lesions which are most interesting. Severe sensorineural deafness is a constant feature of Pendred syndrome, but early reports of imaging of the petrous temporal bone showed no abnormality. However an histological temporal bone study showed both Mondini cochlea and large vestibular aqueduct anomalies (Hvidberg-Hansen and Salslev Jorgensen, 1986).

Subsequent imaging studies confirmed the deficiency of the apex of the interscalar septum of the Mondini type cochlea, but failed to mention the large vestibular aqueduct (Illum et al., 1972; Johnsen et al., 1989). Our studies of a large series of Pendred patients have confirmed the cochlear deformity but not as a convincing feature in every case. This is unlike the enlargement of the vestibular aqueduct and endolymphatic duct and sac which seems to be a constant feature of Pendred syndrome (Figure 1). Moreover some of these cases show areas of low signal on the T2-weighted sections in the region of the sac and duct, suggesting possible fibrosis. It is pertinent that in the original histological description dilated cavities were found in the vestibular part of the membraneous labyrinth partially filled with connective tissue in the sites of the utricle and saccule (Hvidberg-Hansen and Salslev Jorgensen, 1986).

Thus, although imaging assessment of the large vestibular aqueduct would seem valuable only from the prognostic point of view or as a prelude to

From the Royal National Nose Throat and Ear Hospital, London, and the Walsgrave Hospital NHS Trust, Coventry, U.K. 


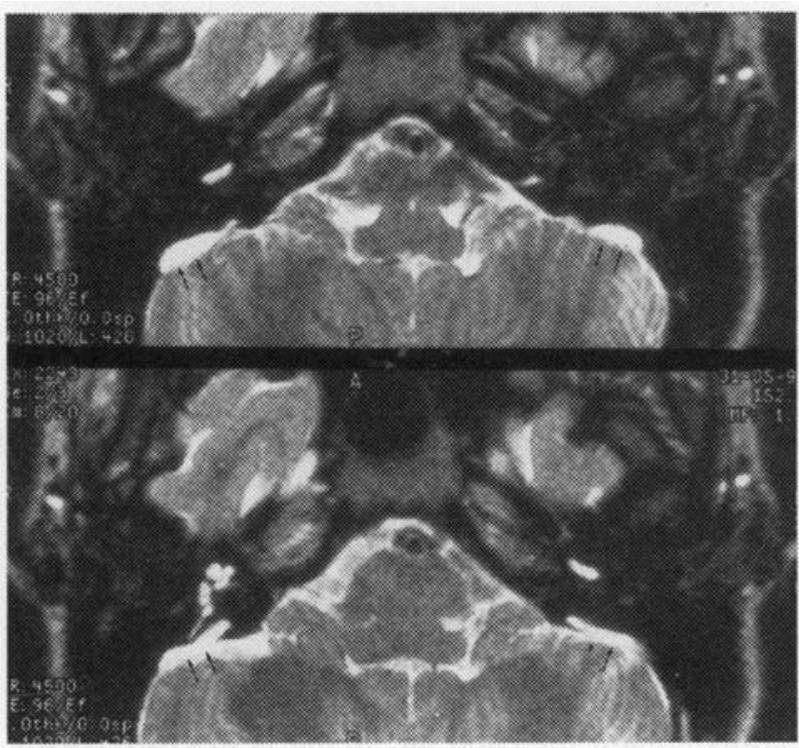

(a)

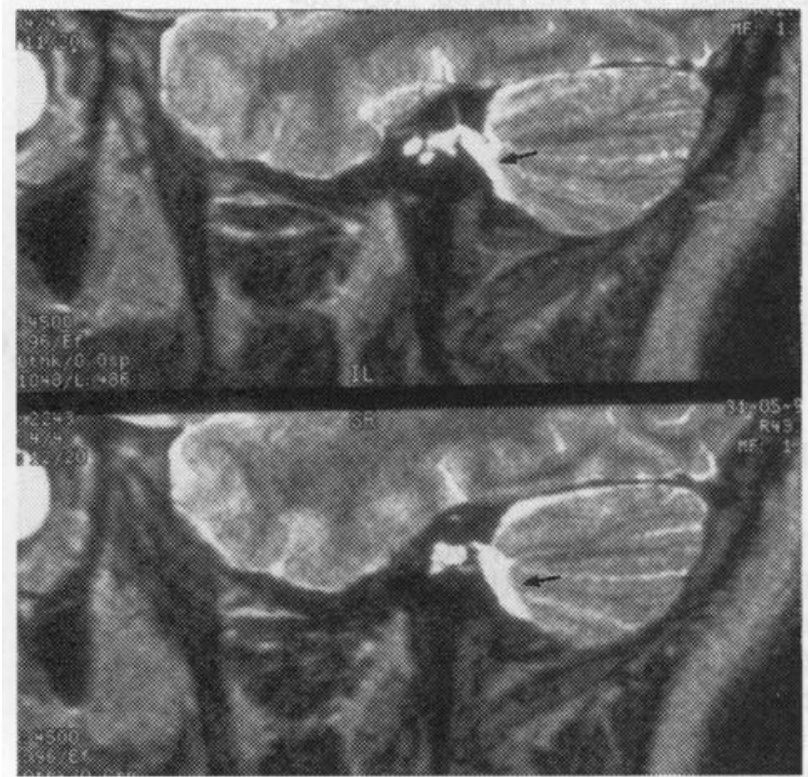

(b)

FIG. 1

Enlargement of the endolymphatic sac and duct bilaterally in a patient with Pendred' syndrome. The vestibular aqueducts are also enlarged; (a) axial; (b) sagittal T2-weighted fast spin echo images.

cochlear implantation (a valuable procedure if the deafness becomes severe), nevertheless MRI with multiplanar capacity and no radiation hazard may well be the initial imaging investigation of choice in those cases of fluctuant and progressive hearing loss associated with a wide vestibular aqueduct. Perhaps in view of the increased cost and more limited availability of high resolution MRI it might be reasonable to screen these patients initially by a few axial CT sections to show whether or not the VA appears wider than normal. In such cases where axial CT does indeed suggest an abnormality of the VA, further examination by MR in axial and sagittal planes would be instigated to assess both the bony outlines of the VA and the state of the endolymphatic sac and duct. The sagittal reformat procedure to assess adequately the diameter of the mid portion of the descending line of the VA can then be abandoned, reducing greatly the radiation hazard (Swartz et al., 1985). Further large series correlating clinical findings with the state of the endolymphatic sac and duct as shown by MR with an outline of the vestibular aqueduct as shown by $\mathrm{CT}$ would seem to be indicated. Does the size of the endolymphatic sac change in relation to changing clinical features? Such assessments are now possible with the new MRI but there now seems to be little indication for attempting surgery to the endolymphatic sac in these congenital disorders any more than for Menière's disease (Thomsen et al., 1996).

Dr. P. D. Phelps,

Royal National Throat, Nose and Ear Hospital, 330 Gray's Inn Road, London WC1X 8DA.

\section{References}

Emmett, J. R. (1985). The large vestibular aqueduct syndrome. American Journal of Otology 6: 387-403.
Gussen, R. (1985) The endolymphatic sac in the Mondini disorder. Archives of Otorhinolaryngology 242: 71-76.

Harnsberger, H. R., Dahlen, R. T., Shelton, C., Gray, S. D., Parkin, J. L. (1995) Advanced techniques in magnetic resonance imaging in the evaluation of the large endolymphatic duct and sac syndrome. Laryngoscope 105: 1037-1042.

Hvidberg-Hansen, J., Salslev Jorgensen, M. (1986) The inner ear in Pendred's syndrome. Acta Otolaryngologica (Stockholm) 102: 239-247.

Illum, P., Kiaer, H. W., Hvidberg-Hansen, J., Sondergaard, G. (1972) Fifteen cases of Pendred's syndrome. Archives of Otolaryngology 96: 297-304.

Jackler, R. K., De La Cruz, A. (1989) The large vestibular aqueduct syndrome. Laryngoscope 99: 1238-1243.

Johnsen, T., Videbaek, H., Olsen, K. P. (1989) CT scanning of the cochlea in Pendred's syndrome. Clinical Otolaryngology 14: 389-393.

Kodama, A., Sando, I. (1982) Dimensional anatomy of the vestibular aqueduct and the endolymphatic sac in human temporal bone. Annals of Otology, Rhinology and Laryngology 91(Suppl 96): 13-20.

Levenson, M. J., Parisier, S. C., Jacobs, M., Edelstein, D. R. (1989) The large vestibular aqueduct syndrome in children. Archives of Otolaryngology, Head and Neck Surgery 115: 54-58.

Mondini, C. (1791) Anatomica surdi nati sectio, Bononiensi scientarium et artium instituto atque academia commentarii. Bonaniae VII; 419-428.

Pendred, V. (1896) Deaf mutism and goitre. Lancet ii: 532.

Phelps, P. D. (1994) Editorial; Dilatation of the vestibular aqueduct/the association with congenital deafness. Clinical Otolaryngology 19: 93-94.

Reussner, L. A., Dutcher, P. O., House, W. F. (1995) Large vestibular aqueduct syndrome with massive endolymphatic sacs. Case Report, ed. Martinez S. A. Otolaryngology Head and Neck Surgery 113: 606-610.

Swartz, J. D., Yussen, P. S., Mandell, D. W., Mikaelian, D. O., Berger, A.-S., Wolfson, R. J. (1985) The vestibular aqueduct syndrome: computed tomographic appearance. Clinical Radiology 36: 241-243.

Thomsen, J., Kerr, A., Bretlau, P., Olsson, J., Tos, M. (1996) Endolymphatic sac surgery: why we do not do it. The nonspecific effect of sac surgery. Clinical Otolaryngology 3: 208-211.

Valvassori, G. E., Clemis, J. D. (1978) The large vestibular aqueduct syndrome. Laryngoscope 88: 723-728. 